\title{
Experience in commissioning the CMS Silicon Tracker and readiness for LHC beams
}

\author{
Joanne Cole* ${ }^{\dagger}$ \\ University of Kansas \\ E-mail: joanne.cole@cern.ch
}

The CMS Silicon Strip Tracker is the largest device of its type ever built. The active area of the strip tracker is approximately $200 \mathrm{~m}^{2}$ and consists of some 9.6 million channels. After a short introduction, the procedures needed to commission the tracker will be described. The first round of commissioning underground took place during June 2008 and some preliminary results from this period will be presented. Finally, the strategy for completing the commissioning procedure will be discussed.

17th International Workshop on Vertex detectors

July 28-August 1, 2008

Utö Island, Sweden

*Speaker.

${ }^{\dagger}$ On behalf of the CMS Strip Tracker Collaboration 


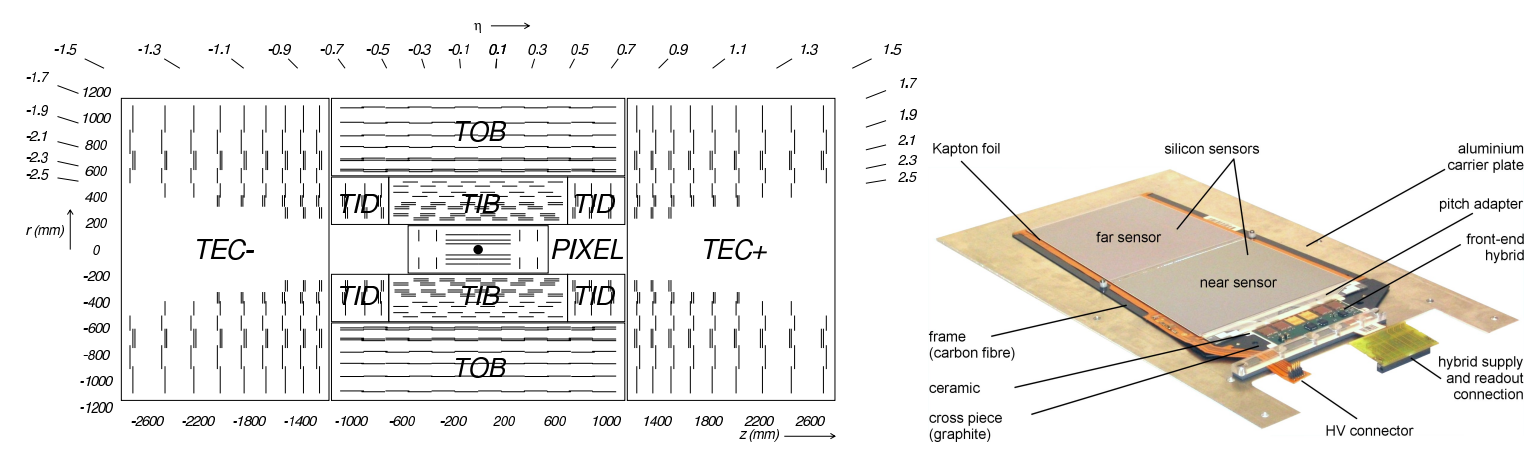

Figure 1: Left: Cross section of the CMS inner tracking system. Right: An example of an SST module mounted on its carrier plate. This specific example is a module from TEC layer 6.

\section{Introduction}

The inner tracking system of the CMS detector is designed to make precision measurements of charged particles produced in $p p$ collisions at the LHC. The tracking system is the closest CMS subsystem to the interaction point, measuring $5.4 \mathrm{~m}$ in length and $2.4 \mathrm{~m}$ in diameter. In order to handle the $40 \mathrm{MHz}$ bunch crossing rate and the relatively high radiation levels in the LHC, a detector technology that offers high granularity and readout speed is essential. These factors have led to the choice of silicon for the entire inner tracking system, making the CMS tracker the largest silicon detector ever constructed. The tracking system consists of two parts: Silicon pixel detectors close to the interaction point and silicon micro-strip detectors starting from a radius of $25 \mathrm{~cm}$. Only the latter will be discussed here; the pixel system is discussed elsewhere [1].

The silicon micro-strip tracker (SST) is divided into four subsystems, as shown in figure 1 (left). The central region is covered by the tracker inner barrel $(25<r<50 \mathrm{~cm})$, TIB, which is composed of four layers and the outer barrel, TOB, which is composed of six layers. The forward regions are covered by the tracker inner disks (TID), consisting of three layers and the endcaps (TEC), which are made up of nine layers. These four subsystems add up to $198 \mathrm{~m}^{2}$ of active silicon area and correspond to approximately 9.6 million channels.

All the subsystems are made up of modules, where a module consists of one or two silicon wafers, plus the on-detector readout electronics, mounted on a carbon fibre or graphite frame. An example from TEC is shown in figure 1 (right). There are 27 different types of module and 15148 modules in total in the four subsystems. The silicon strip sensor pitch ranges from $80 \mu \mathrm{m}$ to $205 \mu \mathrm{m}$. This variation in pitch is needed to handle the expected occupancies and to ensure good two-hit resolution throughout the SST.

Signals are collected every $25 \mathrm{~ns}$ from the strips and are stored using the on-detector readout electronics, namely the APV25 front-end chips [2]. The APV consists of a pre-amplifier, a $50 \mathrm{~ns}$ CR-RC shaper and a 192-element analogue pipeline, in which the signal samples may be stored for up to $4.8 \mu \mathrm{s}$. Each APV is capable of receiving data from 128 strips. On receipt of an external trigger (the tracker has not been designed to trigger itself), the signal is either output as is ("peak" mode), or it can be passed through a deconvolution circuit that reduces the signal width by using the weighted sum of three adjacent cells ("deconvolution" mode). The latter is mandatory for running 

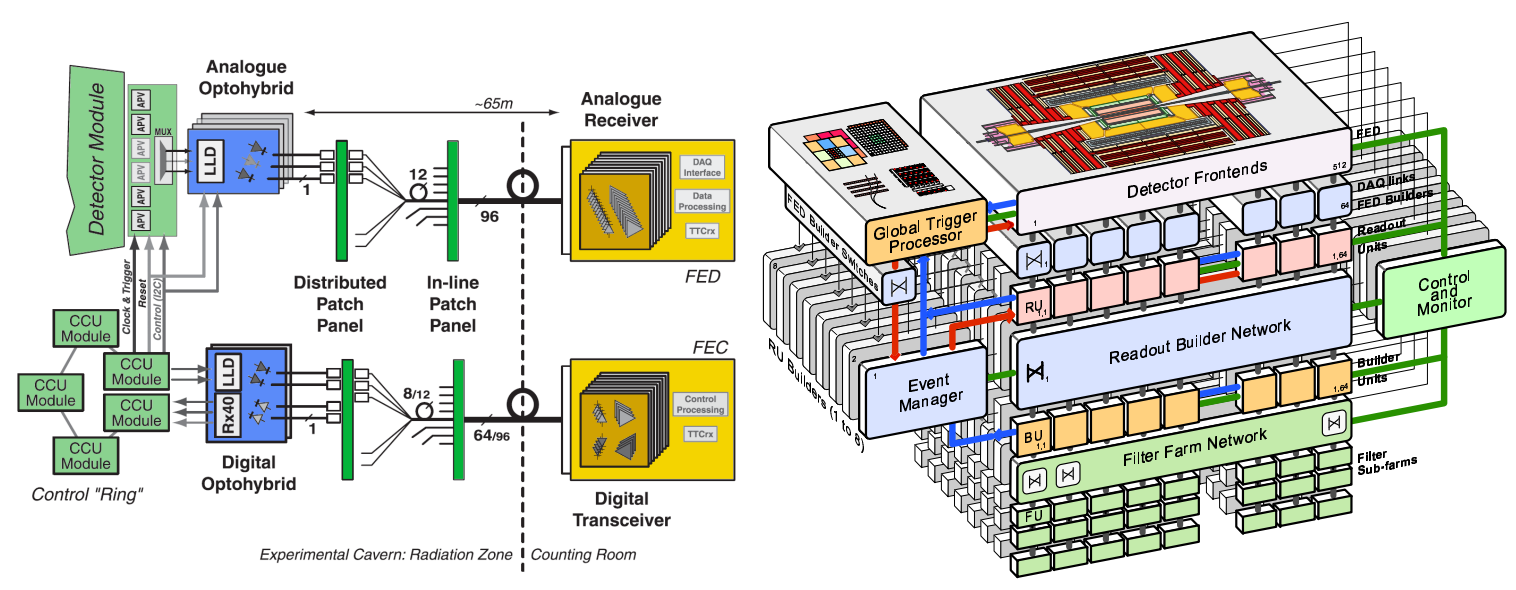

Figure 2: Left: Schematic of the SST readout and control system. Right: Schematic of the CMS central data acquisition system, also used standalone for "local" SST running.

at design luminosity. The data is then sent via optical links to the Front End Drivers (FED) [3], where the analogue-to-digital conversion of the data is performed. In total, 440 FEDs are required to readout the full SST.

\section{The CMS Tracker readout and Data Acquisition}

The control system is responsible for the distribution of slow control commands, clock and trigger signals to the SST. It is made up of 300 so-called "control rings", each of which is controlled by a Front End Controller (FEC) [4]. The signals from the FECs are transmitted via optical links to the digital opto-hybrids ( $\mathrm{DOH})$ and from there to the detector modules via the Commumincation and Control Units (CCU). The control system also includes Detector Control Units (DCUs) on both the modules and on the CCUs, an ASIC used for environmental monitoring [5].

The readout system consists of the APVs and the FEDs, which are connected to each other via analogue opto-hybrids ( $\mathrm{AOH})$ and hence, analogue optical fibres. The signals from pairs of APVs are multiplexed together, converted into an analogue optical signal and then sent to a single FED channel. The FEDs receive pulse height data from up to 96 pairs of APVs. They then digitize the data, perform pedestal subtraction and noise suppression, before carrying out cluster finding. When requested, they send the processed data on to the central data acquistion system (CDAQ). The control and readout systems are summarized in figure 2 (left).

The majority of the data needed for SST commissioning is taken in so-called "local" mode, that is, taking data independent of the rest of the experiment. In order to take data in local mode, a copy of the CDAQ system must be run on SST computing facilities. The CDAQ system is summarized in figure 2 (right). The readout units (RU) construct full events from the fragments received from all the SST FEDs. Once the events have been constructed, they are processed by the builder/filter units (BUFU) for use in data quality monitoring and in the Higher Level Trigger. In local mode, the BUFU performs real-time analysis of the commissioning data and write the results out to disk in ROOT files [6]. The raw data are written out separately to disk in standard CMS data format. 


\section{From integration to commissioning}

Extensive experience has already been gained in running the SST and its associated electronics, prior to the installation of the SST underground in CMS in December 2007. The first opportunity was during summer 2006, when a small prototype of the SST, made up of samples of the different module types, was included in the Magnet Test Cosmic Challenge [7]. This was the first attempt to run as many CMS subdetectors together as possible in the presence of the full magnetic field. Subsequently, the integration of the SST proper started in November 2006 in the Tracker Integration Facility (TIF) at CERN. This culminated in several months of cosmic data-taking with about $15 \%$ of the SST at a range of different temperatures from room temperature down to $-15^{\circ} \mathrm{C}$ coolant temperature. This so-called "Slice Test" [8] was particularly useful for gaining operational and commissioning experience with both the final SST and the final read-out electronics. The 5 million cosmic events that were accumulated also provided valuable information for understanding detector behaviour, track reconstruction and alignment.

The installation of the SST underground was followed by the connection of SST services in the first few months of 2008. Towards the end of this period, it was possible to perform the first tests of the commissioning procedures using temporary cooling. The final cooling system became available in early June 2008, after which the first round of SST checkout and commissioning was performed. The goal of this first round was to allow the SST to participate in a period of cosmic data-taking with other CMS subdetectors in early July ("global run") - the first time the SST was to take data underground. The results of the first round of commissioning are discussed in the following sections.

\section{CMS Tracker commissioning procedures}

Clearly, before any kind of commissioning procedure can be performed, it must been ensured that the SST has cooling, power and safety interlocks. These are controlled by the Tracker Safety and Control systems. The tracker safety system is based on Programmable Logic Controllers (PLC), which provide hardware interlocks in case of problems such as loss of cooling, HV trips etc. The tracker control system is based on PVSS software [9], through which the environmental conditions of the SST may be monitored and the SST powered on and off. The control system attempts to power off the SST in the event of problems, before the hardware interlocks from the safety system kick in.

Once the SST is powered and cooled, the procedures described below can be performed. They are divided into "checkout" and "commissioning" procedures, although the checkout procedures also form part of the commissioning sequence. Checkout procedures provide information about any missing, swapped or poor quality connections. The procedures that are only included in the commissioning category are purely for optimising the performance of the SST and do not provide any new information about SST connections.

\section{Checkout procedures}

DCU-PSU map: A scan is performed to map the control system to the power supply system via DCUs on the detector modules. This allows any electrical connections that were missed or 
swapped to be identified.

DCU-Readout map: This is two-step procedure. The first step is a simple scan of all control system devices, followed by a scan of all readout system devices. This ensures that, as well as all the expected devices being present, all the devices are accessible for further studies. Once all the devices have been successfully identified, the front-end laser devices that drive the optical links are used to send unique patterns to each corresponding FED channel. This allows all connections to be established and any misconnected or poorly connected optical links to be identified.

Internal timing scan: This is the first data-based procedure, in which a scan of the timing of all SST channels is performed so that they can be synchronized to one another. The synchronization is achieved by applying timing delays to the modules via the programmable Phase Lock Loop (PLL) chips installed on each front-end hybrid for this purpose.

Optical gain scan: Once the SST has been synchronized, the optimal bias and gain settings for the front-end laser devices are determined.

Analogue baseline tuning: A second internal timing scan is performed after the optical gain scan. Then the so-called "VPSP" parameter on all the APVs is optimized. This procedure essentially finds the optimal point in the dynamic range of the APV for the pedestals.

Pedestals and noise: This is the final checkout procedure in which the level of pedestals and noise for each APV are determined. It is performed twice, once with only the low voltage supplies enabled and once with both low and high volts switched on. The noise levels change depending on whether the high volts were on or not and therefore a comparison of the two sets of results provides information about faulty high voltage channels.

\section{Commissioning procedures}

APV pulse shape tuning: This procedure tunes the APV pulse shape to ensures that it has the design characteristics. This leads to a uniform detector response over the full SST and promotes long-term stability.

Calibration pulse measurement: This procedure is used for offline calibration and detailed response studies.

APV latency scan: This procedure requires an external trigger (provided in that period by the CMS Drift Tube muon chambers), so as to synchronize the SST with the LHC clock. This essentially means timing the SST in to the level of the $25 \mathrm{~ns}$ bunch crossing time of the LHC.

Fine tuning of pulse shape sampling: This final procedure tunes the timing of the SST down to less than $1 \mathrm{~ns}$, by correcting for any remaining timing effects, such as time-of-flight. 


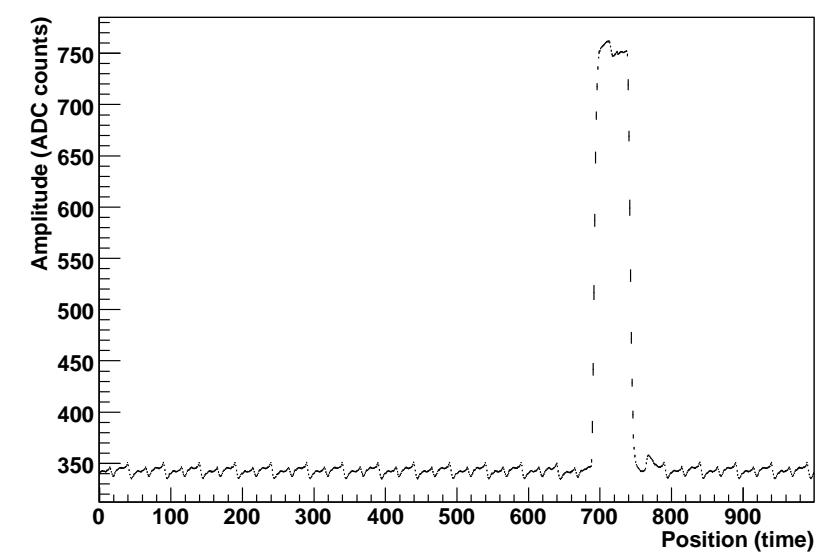

Figure 3: An example of a tick mark observed in the SST during the internal timing scan.

\section{Preliminary results from Tracker commissioning}

The results presented in this section were determined during the first round of commissioning of the SST and are therefore preliminary. A huge amount of data was accumulated in this first round, so it would be impossible to show it all here. Instead, a selection of results are shown to demonstrate some of the commissioning procedures and to indicate the quality of the SST.

The aim of the first round of commissioning was to validate the commissioning procedures on a large-scale system and to get the SST ready for inclusion in the global run scheduled for early July 2008. A deliberately conservative approach was taken, ie. anything that was not completely understood was excluded. The second round of commissioning has since been completed, in which a systematic study was made of all the channels excluded from the first round. The second round was also used for large-scale testing of all the commissioning procedures that were not tested during the first round.

The internal timing scan is the first data-related commissioning procedure [10]. When receiving a clock signal but no triggers, the APVs generate synchronization pulses, known as "tick marks", every $1.75 \mu$ s (see figure 3 ). These tick marks can be used to internally synchronize all channels. The tick marks are synchronized such that they all match the "last" tick mark. The optical fibres in the readout system are used during the internal timing scan. The lengths of these fibres are known and therefore the corresponding delays can be calculated. To ensure that the readout fibres do not affect the results of the scan, the fibre length corrections are applied beforehand. The remaining differences between the tick mark positions that are corrected for by the internal timing scan arise from the relative position of each APV in its respective control ring and the different lengths of the optical fibres in the control system.

The results from the timing scan performed for TIB/TID are shown in figure 4. The delays that must be applied in the PLLs, as determined during the timing scan, are shown as a function of APV number for TIB and TID. It is clear that the spread of the delay values is relatively large, indicating why this procedure is so important. As this procedure relies on seeing good tick marks 


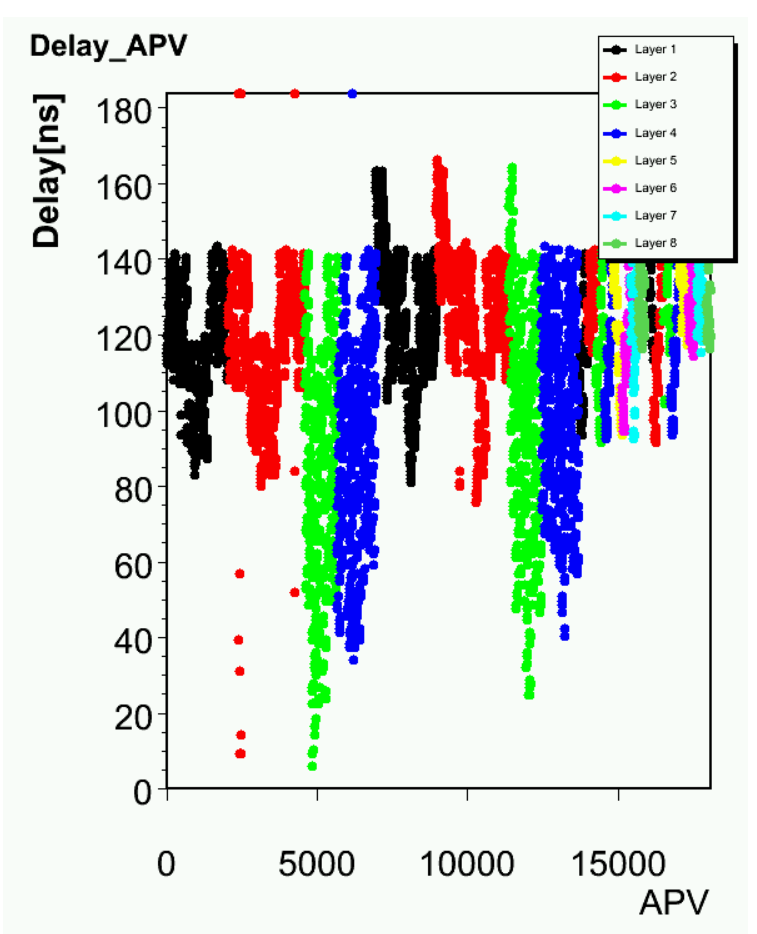

Figure 4: The delays implemented in the PLLs as a function of APV number for TIB and TID, subdivided by layer, as determined in the internal timing scan. The spread of values reflects the different locations of the APVs on their respective control rings, as well as the different length connections between the APVs and the DOHs.

from all APVs, any problems with the tick mark can be identified by this procedure and the affected modules flagged for further investigation.

The optical gain scan optimizes the use of the available dynamic range of the AOH by scanning the laser bias values for different gain settings, whilst measuring the tick mark amplitude (the digital 1 level). As can be seen in figure 5 the laser diode output scales linearly with input current above a certain threshold. The slope of the scaling, as well as the threshold are monitored. The left-most curve corresponds to the top of the tick mark, while the right-most curve corresponds to the tick mark baseline as a function of bias setting. By comparing the two curves, the output tick mark seen at the FED and the estimated input tick mark to the linear laser driver can also be monitored.

Pedestal and noise data need to be taken regularly, as they are needed for the zero-suppression algorithm in the FED. They are also needed for clustering, which is used in the Higher Level Trigger as well as in offline reconstruction. During the slice test at the TIF, it was established that the pedestals are very stable with respect to time, for a given coolant temperature. This observation has subsequently been confirmed with the SST installed within CMS. During the first round of commissioning, the quality of the SST was found to be excellent: the number of dead strips is much less than $1 \%$, while the number of noisy strips is less than $1 \%$. The mean noise is currently substantially less than 2000 electrons per strip, as intended for unirradiated sensors [11].

In order to be able to take data with the rest of the detector, it is only mandatory to perform 


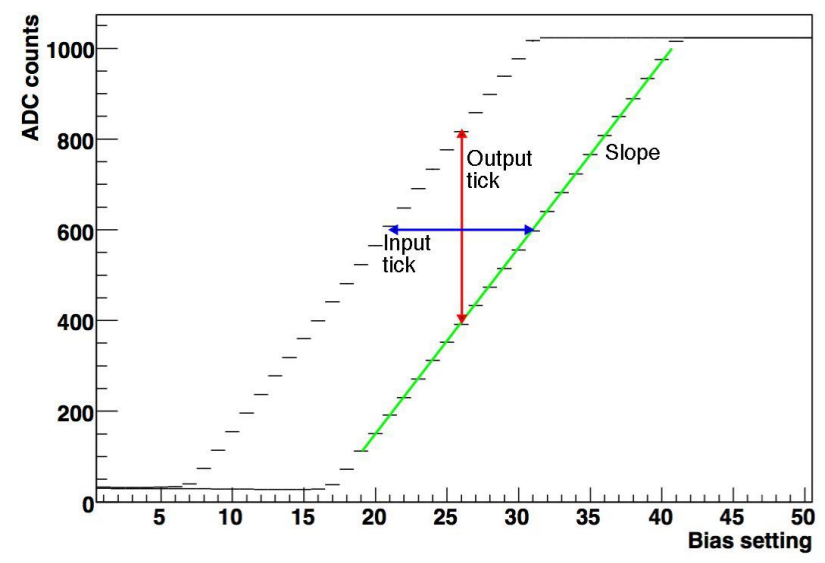

Figure 5: The quantities monitored in the optical gain scan. The left-most curve corresponds to the top of the tick mark, while the other curve corresponds to the baseline as a function of bias setting for a particular gain setting. The scaling of the laser diode output and the thresholds are also visible.

the checkout procedures and the APV latency scan. Under these circumstances the SST will work, but the performance will not be optimal. Given the limited amount of time available for the first round of commissioning, it was only possible to perform this minimal set of procedures. As a consequence it is only possible to show results from one purely commissioning procdure, ie. the APV latency scan.

The absolute synchronization of the SST was determined using an external trigger provided by the CMS drift tube muon chambers. The APV latency scan is performed in steps of $25 \mathrm{~ns}$ (the bunch crossing time of the LHC) and the optimal latency setting is determined by reconstructing the pulse shape. The optimal working point is then determined by fitting the pulse shape distribution and finding the peak value. Figure 6 shows the fitted distributions for TID, TIB and TOB. These are, once again, the results from the first round of commissioning and as such, are based on limited statistics. The optimum values for the three subdetectors are broadly consistent.

The first round of commissioning was successfully completed in time to allow TIB, TID and TOB to participate in the global run in early July 2008. TEC+ was also included part way through the run. TEC- was excluded because it was not possible to tune the parameters of the cooling system sufficiently for this subdetector to ensure stable running in time for the global run. Even though the strategy for the first round of commissioning was deliberately very conservative, the quality of the connections was found to be excellent, as can be seen in table 1. A successfully checked-out module is one that has passed all checkout procedures, without any indication of problems.

\section{Conclusions}

The CMS silicon strip tracker has been successfully completed and installed underground. The commissioning procedures that were developed during the "Slice test" at CERN, were validated when the SST was running on temporary cooling in spring 2008. The first round of commissioning with the final cooling plant began in June 2008 and was completed in time for the SST to participate 

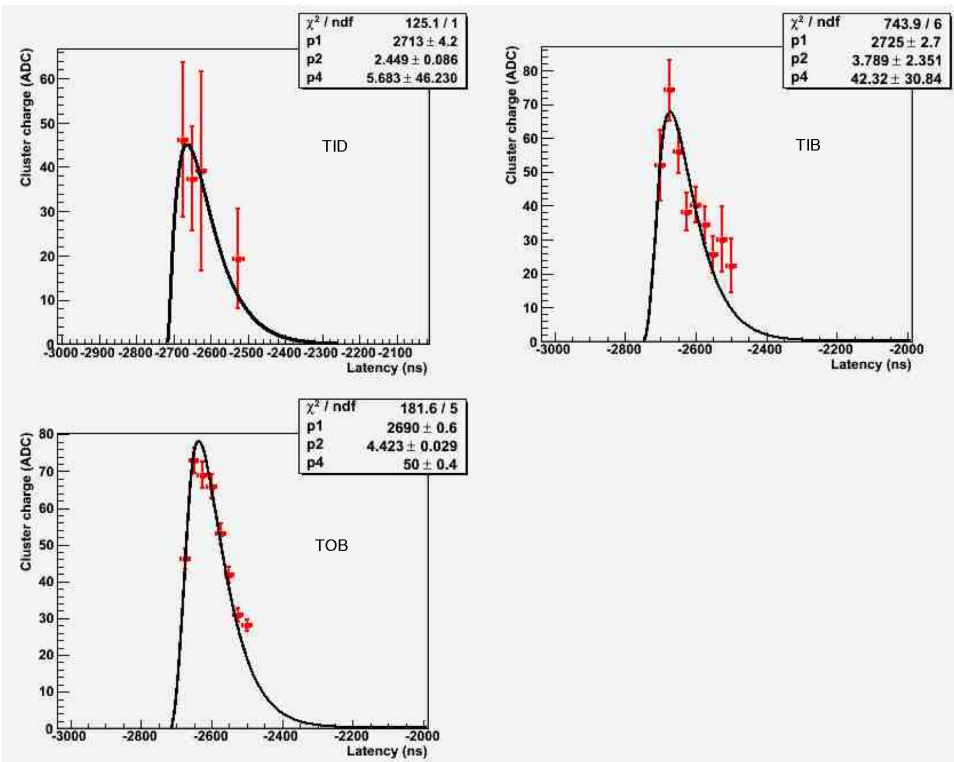

Figure 6: The fitted pulse shape distributions for TIB, TID and TOB, as observed in the APV latency scan. The fit allows the peak of the distribution to be determined. The closest latency setting to the peak of each distribution is taken as the latency setting for each subdetector.

\begin{tabular}{|c|c|}
\hline \hline Subdetector & Fraction of successfully checked out modules \\
\hline TIB/TID & $97 \%$ \\
TOB & $97 \%$ \\
TEC+ & $95 \%$ \\
TEC- (partial) & $94 \%$ \\
\hline \hline
\end{tabular}

Table 1: A summary of the percentage of modules successfully checked out and commissioned during the first round of SST commissioning. TIB, TID and TOB participated fully in the July global run, while TEC+ joined part way through. TEC- did not participate due to cooling issues (see text).

in cosmic data-taking with other subdetectors - the global run - in early July. The strategy for the first round of checkout and commissioning was to be deliberately very conservative: any modules that were not completely understood were set aside for further investigation and not enabled during the global run. The quality of the SST was found to be excellent: 95 - 97\% of the SST barrel was included in the global run, while $95 \%$ of the positive endcap joined part way through the run. The negative endcap did not participate, as it was not possible to tune the cooling system settings for this subdetector in time.

A lot of operational experience was gained during the first round of commissioning and many aspects of the SST performance were understood. The quality of the SST was found to be very good. The main issue during the first round was the reliability of the cooling plant, which has had a number of problems. Many of these problems have been solved and the second round of commissioning has subsequently been completed. The SST has performed well in recent cosmic 
data-taking with the CMS magnet at full field.

\section{References}

[1] A. Ryd, CMS pixel experience in detector commissioning, in the proceedings of the 17th International Workshop on Vertex detectors, POS (VERTEX 2008) 014

[2] M.J. French et al., Design and results from the APV25, a deep sub-micron CMOS front-end chip for the CMS tracker, Nucl. Instr. Meth. Phys. Res. A466 (2001) 359-365.

[3] C. Foudas et al., The CMS tracker readout front end driver, IEEE Trans. Nucl. Sci. 52 (2005) 2836-2840.

[4] F. Drouhin et al., The CERN CMS Silicon Strip Tracker Control System, CERN-CMS-CR-2004-032.

[5] G. Maguzzu, A. Marchioro and P.Moreira, The Detector Control Unit: An ASIC for the monitoring of the CMS tracker, Nuclear Science Symposium Conference Record 2003 IEEE, Vol. 2 (2001) 1206-1209.

[6] R. Brun and F. Rademakers, ROOT: An Object-Orientated Data Analysis Framework, http://root.cern.ch.

[7] D. Abbaneo et al., Tracker Operation and Performance at the Magnet Test and Cosmic Challenge, CERN-CMS-NOTE-2007-029.

[8] W. Adam et al., Silicon Strip Tracker Detector Performance with Cosmic Ray Data at the Tracker Integration Facility, CERN-CMS-NOTE-2008-032.

[9] PVSS made by ETM Professional Control AG, Eisenstadt, Austria, http://www.pvss.com.

[10] K. Gill et al., Synchronization of the CMS Tracker, in the proceedings of the 9th Workshop on Electronics for LHC Experiments, CERN-LHCC-2003-055.

[11] CMS Collaboration, The Tracker Project Technical Design Report, CERN-LHCC-1998-006. CMS TDR 5, Addendum CERN-LHCC-2006-016. 